manned by professionals with clear duty and responsibility to provide comprehensive care for those in need. It is my opinion that in large part problems have occurred because the Consultant is not accepted as the leader of his clinical team. Another factor would appear to be confusion, both clinical and legal. The patient is seen only in terms of what his behaviour was, not how his clinical condition presents now. This is further compounded by a semantic game whereby the chronic schizophrenic of 15 to 20 years standing becomes 'a psychopath' and therefore no-one's responsibility. The application of Section 65 to the Hospital Order does not require the hospital or the Responsible
Medical Officer to provide 'Security'. Further Home Office involvement does not in fact impede clinical freedom, but merely underlines the element of responsibility. My plea is for extended professional contact to restore mutual trust and confidence so that we do, in fact, continue to provide a National Health Service. Paraphrasing Prospero:

'For these here confined by you

Let your indulgence set them free'.

The views expressed in this paper are those of the author and do not necessarily reflect those of the Department of Health and Social Security.

\title{
Trends in Length of Stay at Broadmoor
}

\author{
by David Tidmarsh, Consultant Psychiatrist, Broadmoor Hospital
}

Little information is available about how long patients stay in the Special Hospitals. This paper describes what is believed to be the first systematic study of this topic.

The inquiry started when I was looking into how best to monitor the overall success and failure rates of treatment at Broadmoor. Because the hospital deals with a long-stay population of patients, whose relapses into serious violence after leaving the hospital are happily rare and often long delayed, it was felt essential to start with the largest possible sample, and this meant starting with patients admitted many years ago. Though a great deal of data on these patients is to be found in their case notes, it would not have been possible to extract this with our present resources. The only alternative source of information available at the beginning of the study was the hospital's admission and discharge registers. These give for each day of the year the names of the patients admitted and discharged. Included are each patient's hospital number, sex, reference to previous admissions, the method of separation* and the address to which he or she went. As it happens, the results of a hospital census taken in 1960 were also available from which, without too much trouble, it was possible to discover who was in the hospital on 1 January 1960 a very convenient starting point shortly before the implementation of the 1959 Mental Health Act. These two sources of data thus made it possible to identify for follow-up purposes all patients in the hospital on 1 January 1960 and all patients admitted and leaving thereafter. It was at this point realized that these meagre data could usefully be analysed to give information about how

* 'Separation' refers to leaving hospital by any method. long these patients stayed, and it is this analysis which is reported here.

Using safeguards about confidentiality similar to those used by the Special Hospitals Research Unit's Case Register, the hospital registers were transcribed and information about each patient then coded onto unidentifiable data sheets and punched onto cards. The data were analysed with the help of an SPSS programme written by myself which made it possible to make a complete reconstruction of the patient population from 1 January 1960 up to 31 December 1979. From this it was possible to select admission and discharge cohorts and also to identify census population at any convenient date and to calculate periods between various events. Errors have not been entirely eliminated, though more obvious ones have been removed after simple inspection of a computer print-out of the data, and others by inspecting basic tabulations. There remains a discrepancy of less than $\frac{1}{2}$ per cent between the statistics generated by the computer and the hospital's statistics compiled manually. These errors do not significantly affect the present results and will be corrected in due course.

\section{Results}

Admissions to Broadmoor almost trebled in the five years after the implementation of the Mental Health Act; they then remained at the level of about 150 patients a year. But since 1971 there has been a steady fall almost back to the figure for 1960 .

The proportion of each year's admission cohort discharged in a given period has changed remarkably little over the last 20 years. Though the proportion leaving within five 
years rose from about 50 per cent for those admitted in 1960 to about 70 per cent for those admitted in 1968 and has since fallen again, there has been no change in the number leaving under 10 years. Whether this constant rate reflects tradition, administrative factors, a lack of improvement in the prognosis of schizophrenia, a constant rate of maturation of personality disordered patients, or the way dangerousness is perceived, cannot be deduced from this study. About 8 per cent of the patients admitted between 1960 and 1964 are still in the hospital and these form a nucleus of 'new chronics'.

The resident population has declined, the males from 712 on 31 December 1959 to 599 on 31 December 1979, and the females from 173 to 121 . These reductions have gone some way to relieve overcrowding and have been caused by the transfer or discharge of long-stay patients. The proportion of male patients who had been in the hospital for over 15 years fell in this period from 24 per cent to 7 per cent, in absolute terms from 172 to 75 patients while the proportion of longstay female patients fell from 39 per cent to 10 per cent, in absolute terms from 67 to 12 patients. The proportion of short-stay patients in the resident population increased until 1974 but is now falling again, reflecting the increasing difficulty in discharging patients. For males the mean 'hospital age' of the resident population was 7.7 years in 1959, fell to a minimum of 5.1 in 1969 and is now up to 6.5 . For females it was 9.2 years in 1959, 5.4 in 1974 and 6.7 in 1979.

After a rapid rise in the early 1960 s to about 150 patients a year, the number of separations, excluding those transferred to other Special Hospitals, has fallen steadily over the last 10 years to only 64 patients in 1979 , a figure lower than that for 1960. This declining rate of separation has had an effect not only on the length of stay of the resident population but also on those who leave. Male patients leaving in the early 1960 s had stayed about eight years. By the early 1970 s they had stayed about five years but now their stay is up again to 6.5 years.

I hope to investigate what effect such variables as age, offence and diagnosis have on length of stay, but at present these data cannot be utilized. Available data however make it possible to assess whether the method of separation has any bearing on length of stay. For this purpose, because some methods are infrequent, the patients who had left were divided into those who had left in the four five-year periods starting with that from 1960 to 1964 . It was found that there was little variation in the rank order of the various methods over these periods. The quickest method, available to only a small minority, was return to prison, the mean length of stay varying between two and three years. The next quickest was repatriation and absolute discharge, again only open to a few patients, with means varying between three and five years. Conditional discharge took between four and eight years. Transfer to conventional NHS psychiatric hospitals varied over these periods between six and ten years, and those who died had been patients in Broadmoor between $7 \frac{1}{2}$ and 10 years.

Transfers to conventional psychiatric hospitals account for half of all Broadmoor's separations. This channel is far and away the most important, and over the last twenty years has been the most common for patients of all 'hospital ages'. Thus 41 per cent of those leaving under five years are transferred, and this proportion rises to 80 per cent of those leaving from 15 to 19 years after admission. Any delay in the process of transfer or any reduction in the numbers transferred will have a major effect on the lengths of stay of our patients, and this is now happening. Between 1965 and 1969 an average of 74 patients were transferred each year. Since then there has been a steady fall to only 30 patients in 1979.

\section{Conclusion}

It is not possible to draw many conclusions from what is essentially a descriptive study based on meagre data, but I would like to make two points. The first is that an interesting comparison can be made between these Broadmoor data and those given by Steadman and Cocozza for the maximum security hospitals in New York State. These authors found that before the changes initiated by the Baxstrom decision the average stay of patients in those hospitais was no less than 22 years. After it the patients who were transferred as a result had stayed 15 years. At Broadmoor the mean length of stay for all separations between 1 January 1960 and 31 December 1979 was only 6.27 years. Clearly, for this reason alone, any comparison of the results of follow-up studies could be very misleading. The second point concerns the trends demonstrated by this analysis. Five years after the implementation of the 1959 Mental Health Act, Broadmoor's turnover had trebled. There was then a plateau, but in the last five years there has been a steep fall in turnover to the 1960 level. This, as Susanne Dell (1980) has shown, has to an important extent been due to a hardening of the attitudes of the psychiatric services to Special Hospital patients. The present study demonstrates that Broadmoor's patients are now staying longer in conditions of maximum security than their predecessors, and it is the firm opinion of Broadmoor consultants that this is unjustifiable on clinical grounds.

\section{REFERENCES}

DeLL, S. (1980) Transfer of Special Hospital patients to the NHS. British Journal of Psychiatry, 136, 222-34.

Steadman, H. J. and Cocozza, J. J. (1974) Careers of the Criminally Insane. Massachusetts: Lexington Books.

The views presented in this paper are those of the author and do not necessarily reflect those of the Department of Health and Social Security. 